\title{
SOA-based Integration of the Internet of Things in Enterprise Services
}

\author{
Patrik Spiess ${ }^{1}$, Stamatis Karnouskos ${ }^{1}$, Dominique Guinard ${ }^{1,2}$, Domnic Savio ${ }^{1}$, \\ Oliver Baecker ${ }^{1,3}$, Luciana Moreira Sá de Souza ${ }^{1}$, and Vlad Trifa ${ }^{1,2}$ \\ ${ }^{1}$ SAP Research, ${ }^{2}$ ETH Zurich, ${ }^{3}$ University of St. Gallen \\ Email: patrik.spiess@sap.com
}

\begin{abstract}
Advances in the areas of embedded systems, computing, and networking are leading to an infrastructure composed of millions of heterogeneous devices. These devices will not simply convey information but process it in transit, connect peer to peer, and form advanced collaborations. This "Internet of Things" infrastructure will be strongly integrated with the environment, and its integration with the enterprise systems will not only further blur the line between business IT systems and the real world, but will change the way we design, deploy, and use services. New opportunities can emerge for businesses, which can now closely collaborate with the real world. The work presented here proposes an architecture for an effective integration of the Internet of Things in enterprise services.
\end{abstract}

\section{Motivation and Vision}

Traditionally, the systems of an enterprise controlling physical processes (like manufacturing, logistics execution, energy monitoring, building automation, etc.) use different standards for data and communication than its business software systems. We see a paradigm change as the former breed of systems increasingly adopt the standards of the latter. It is expected that this trend will continue, while in parallel the number of connected devices will explode.

As we are moving towards the "Internet of Things" as depicted by [3], millions of devices will be interconnected, providing and consuming information available on the network and cooperate. As these devices need to interoperate, the service-oriented approach seems to be a promising solution, i.e. each device should offer its functionality as standard services, while in parallel it is possible to discover and invoke new functionality from other services on-demand.

As depicted in Figure 1, future infrastructures will be service-oriented. As such, new functionality will be introduced by combining services in a cross-layer form, i.e. ser-

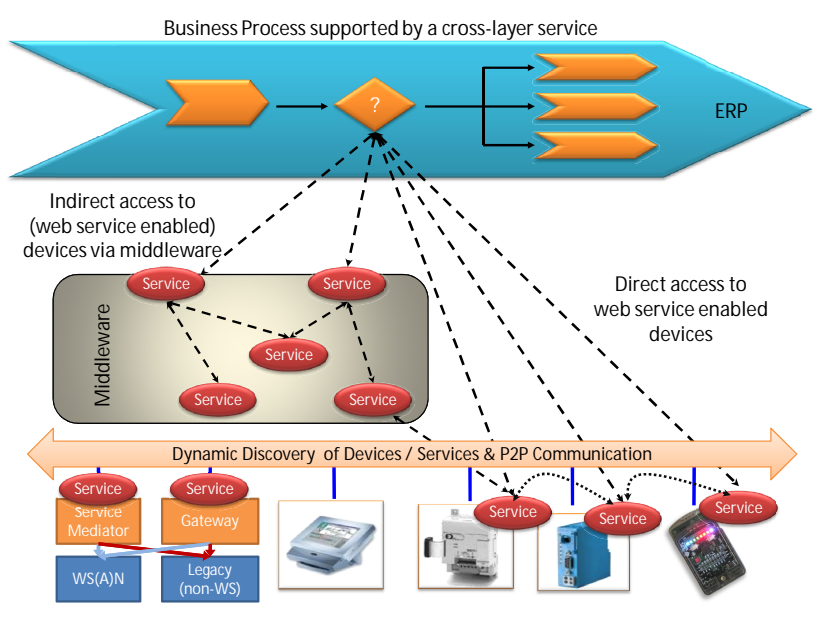

Figure 1. Vision of Web Service Mash-Ups

vices relying on the enterprise system, on the network itself and at device level will be combined. New integration scenarios can be applied by orchestrating the services in scenario-specific ways. In addition, sophisticated services can be created at any layer (even at device layer) taking into account and based only on the provided functionality of other entities that can be provided as a service [5]. In parallel, dynamic discovery [2] and peer-to-peer communication will allow to optimally exploit the functionality of a given device. It is clear that we move away from isolated stand-alone hardware and software solutions towards more cooperative models.

The convergence of solutions and products towards the SOA paradigm adopted for smart embedded devices contributes to the improvement of the reactivity and performance of industrial processes, such as manufacturing, logistics, and others. This will lead to information being available in near real-time based on asynchronous events, and to business-level applications that are able to use high-level information for various purposes, such as diagnostics, performance indicators, traceability, etc. SOA-based vertical 
integration will also help to reduce the cost and effort required to realize a given business scenario as it will not require any device drivers or third-party solutions.

\section{SOCRADES Integration Architecture}

To realize an effective integration of the capabilities offered by the Internet of Things, we have designed an architecture (depicted in Figure 2) that exposes real-world devices with embedded software to standard IT systems by making them accessible in a service-oriented way. The requirements we want to cover as well as the functionality to be realized is analyzed in [6],[1]. The architecture implemented hides the heterogeneity of hardware, software, data formats and communication protocols that is present in today's embedded systems. The specifications foster open and standardized communication via web services at all layers. The following layers can be distinguished: Application Interface, Service Management, Device Management, Security, Platform Abstraction and Devices.

Application Interface: The SOCRADES Integration Architecture (SIA) enables enterprise-level applications to interact with and consume data from a wide range of networked devices using a high-level, abstract interface that features web services standards. Those standards already constitute the standard communication method used by the components of enterprise-level applications. Web services are the technology canonically used to implement business processes, which are frequently modeled as orchestrations of available web services. This allows networked devices that are connected via the middleware presented here to directly participate in business processes while neither requiring the process modeler nor the process execution engine to know about the details of the underlying hardware.

The integration architecture features a messaging system, because the application should be able to consume any events whenever it is ready to and not when a low-level service happens to send them. It also allows the application to interact with devices that are only intermittently connected, receiving notifications when a certain device becomes available again or have the system cache the message and deliver it when the device is ready to receive it. Finally there is a service catalog that users and applications can query. It finds and delivers service descriptions and pointers to running service instances. Both atomic services hosted by the devices and higher-level composed services are listed here.

Service Management: All functionality offered by networked devices is abstracted by services. Either devices offer services directly or their functionality is wrapped into a service representation. On this layer of the integration architecture and on all layers above, the notion of devices is abstracted from and the only visible assets are services. An important insight into the service landscape is to have a

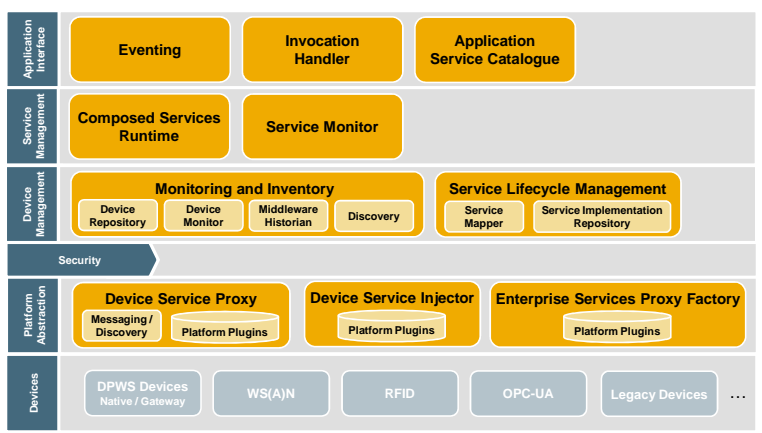

\section{Figure 2. SOCRADES Integration Architec- ture}

repository of all currently connected service instances. This layer also provides a runtime for the execution of composed services. We support the composition of business processes primarily by offering an execution service for underspecified BPEL processes, meaning that service compositions can be modeled as business processes where the involved partners do not need to be explicitly specified at design time. This flexibility is needed since the set of services offered at the device level is dynamic; as (potentially mobile) devices connect and disconnect or are re-configured, the available service set changes frequently. Traditional business process execution languages like BPEL are not built to support such dynamics. With some proposed extensions to BPEL and the execution support for the extended processes, the process modeler has unprecedented flexibility.

Device Management: All devices are dynamically discovered, monitored and their status is available to the enterprise services. Furthermore, it is possible to remotely deploy new services during run-time, in order to satisfy application needs.

Security: Both devices and back-end services may only be accessed by clients that have a certain role and provide correct credentials that authenticate themselves. This layer implements the correct handling of security towards the devices and the enterprise-level applications.

Platform Abstraction: As stated before, devices either offer services directly or their functionality is wrapped into a service representation. This wrapping is actually carried out on the platform abstraction layer. In the best case, a device offers discoverable web services on an IP network. In this case no wrapping is needed because services are available already. If the device type however does not have the notion of a service (it might use a message-based or data-centric communication mechanism), the abstraction into services that offer operations and emit events can be a complex task. In addition to service-enabling the communication with devices, this layer also provides a unified view on remotely installing or updating the software that runs on 


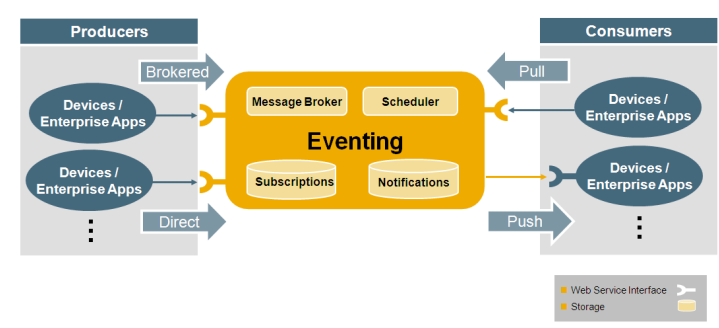

Figure 3. Eventing

devices and enables the devices to communicate natively, i.e. in their own protocol with back-end devices.

Devices: Heterogeneous Devices are expected to connect to the architecture. These include industrial devices, home devices, or IT systems such as mobile phones, PDAs, production machines, robots, building automation systems, cars, sensors and actuators, RFID readers, barcode scanners, or power meters. We used several of the listed types of devices during prototype implementations as shown in the Demonstration section.

\section{Architecture Design}

The architecture components presented in the following subsections have all have been implemented based on open WS-* standards in Java EE 5 on SAP NetWeaver platform, using EJB 3.0 and JPA.

\subsection{Eventing}

This component is an implementation of the WSBrokered Notification standard, implementing publish and subscribe functionality, to distribute information about events of interest. Both Push or Pull type delivery are supported and both involve the role of "Consumer" and "Producer". Figure 3 shows a high-level view on the Eventing component.

The Eventing component acts as an intermediary between a Producer and the entity that has interest in the generated events. For example, a shop floor device could propagate sensor data through the Eventing component acting as a Producer.

The WS-Notification standard consists of 3 specifications: WS-Notification, WS-Brokered Notification, and WSTopic.

WS-Notification defines consumer and producer roles and the interfaces both have to provide. This allows for a point to point event subscription and delivery. The producer needs to provide the notification interface where the producer sends the notifications to and the producer has to provide the subscription interface so that the consumer can subscribe.

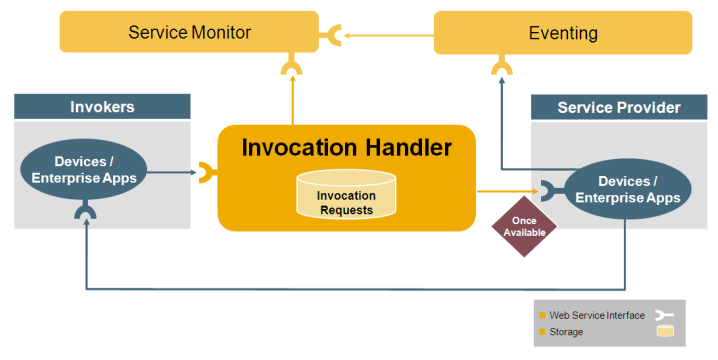

Figure 4. Invocation Handler

WS-Brokered Notification builds on top of WSNotification and specifies an intermediary called notification broker, which is exactly what we have implemented in the Eventing component. It provides the subscription interface on behalf of the producer and the notification interface on behalf of the consumer. This drastically reduces the complexity of consumers and producers and allows flexible relationships, that can involve producers/consumers unknown at design and deployment time. Additionally, the broker offers pull point functionality, i.e. it can collect notification for a consumer until it retrieves them, further reducing the complexity of the consumer.

WS-Topics defines hierarchical topic trees that can be specified and used to declare event types when producing and event filters when subscribing to events. The eventing component implements basic topic expressions, the simplest form of topics specified.

\subsection{Invocation Handler}

The Invocation Handler (Figure 4) implements functionality to act as an intermediary for service invocations. It is capable of buffering an invocation in case an endpoint is not available. Furthermore, it supports two different types of delivery (Direct Service Response and Push) and provides interfaces for the invoker to query for an invocation request status and canceling a pending invocation.

When using the Direct Service Response delivery, the invoker has to supply an endpoint which supports retrieving the service invocation response directly. This endpoint can be the invoker itself or any other supporting entity. The Invocation Handler passes the given endpoint as the return endpoint when invoking the service. The delivery mechanism Direct Service Response is depicted in Figure 4.

When using the Push delivery, the Invocation Handler receives the service invocation response and publishes it to the Eventing component. The invoker has to supply the description of the event that should be used when the response is propagated to the Eventing component. Using the Push delivery implies that the invoker has previously registered interest for the supplied event description, using one 


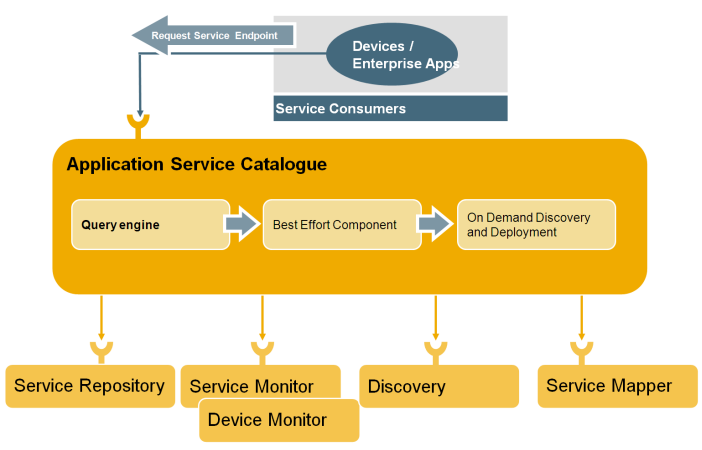

Figure 5. Application Service Catalogue

of the Eventing subscription types (Push/Pull). Therefore the Eventing component is used as an intermediary for delivery of the service invocation response.

The component implements functionality to retrieve the current status of a request for invocation. This is one of "Pending" and "Not Pending". "Pending" implies that the service invocation was not processed, possibly because the service was not yet available. "Not Pending" implies that the service invocation was executed, if the given request for invocation was registered previously. This functionality is used by the invoker to know whether a service invocation happened within a given time or whether there was a conflict that prevented the service to be executed properly. The component implements functionality to remove a request for invocation. The invoker can decide to remove a request in case a response or the invocation of the service would result in unwanted consequences or be of no interest.

\subsection{Application Service Catalogue}

One of our main targets and justification for wanting WS everywhere is the fact that they enable more flexibility. Once every service is exposed using the same interfaces one can really think about combining the services and creating new behaviors for the application. Yet, composition and service consumption does not really make sense if services cannot be searched for. This is the goal of the Application Service Catalogue. Figure 5 depicts its subcomponents. It is the search engine of the architecture that enables the applications to look for services based on queries.

The catalogue is composed of three subcomponents: QueryEngine: The QueryEngine is the core of the search; it consumes a simple text query and returns an ordered list of matching Service Types. In order to go beyond the simple query matching of a service name or description it first uses a concrete instance of a pluggable component called QueryStrategy with the goal to define how the simple query should be extended before processing. The use of a QueryStrategy component prevents from locking the architecture

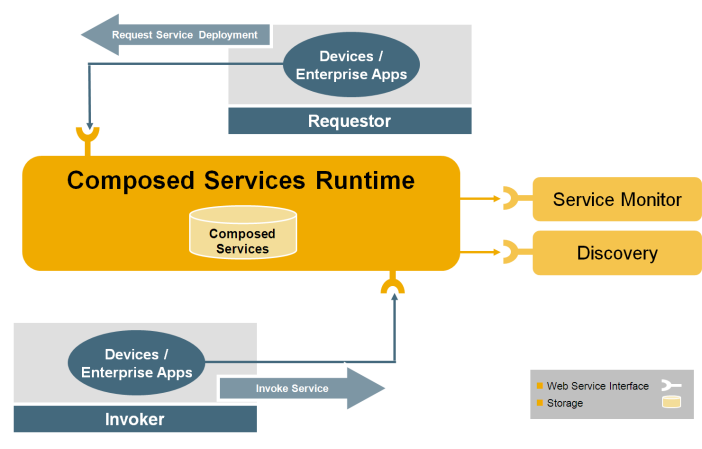

Figure 6. Composed Services Runtime (CSR)

with a search method that is not appropriate.

BestEffortComponent: This is responsible for selecting the best possible device running the selected service. "Best" is here meant in terms of QoS (Quality of Service). It takes into account the information held by the Device Monitor namely, status, battery life, etc.

OnDemandDiscoveryAndDeployment: This component is responsible for initializing the dynamic search for services. When a service type corresponding to the query is found but the Best Effort component does not find a running instance, the On Demand Discovery and Deployment component will try: (i) to force a discovery for such a service, using the Discovery component, and (ii) to inject the service on a device, using the Service Mapper and the Device Service Injector.

\subsection{Composed Services Runtime}

Composed services are described in BPEL, which communicates with service partners over partner links. At design time, all partner links must be bound to concrete service types. Only the actual endpoints can be unknown at design time. CSR can host several composed services, depending on the device capacity, e.g. RAM or CPU power. Moreover, as we need a JVM to run CSR, the performance of CSR also depends on the garbage collector and the Java heap size of that JVM. CSR (depicted in Figure 6) basically provides two interfaces for web service consumers: the interface for deploying/undeploying composed services and the interface for using composed services. For deploying new composed services, CSR hosts a service called Deployment Service. Deploying a composed service on CSR means that a SOAP message is sent to the Deployment Service, containing the URL location of the BPEL description of the new composed service and also the URL location of the deployment descriptor of that service. A new composed service instance is created, hosted on CSR, and represented in the Service Monitor. During the execution of a composed service runtime, CSR asks the Service Monitor for the cost 


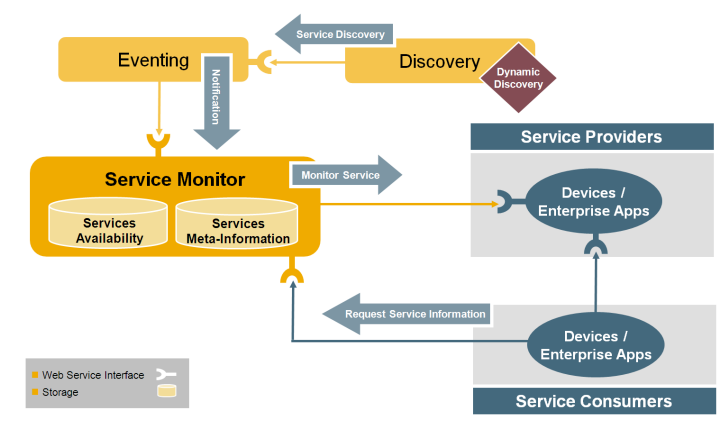

Figure 7. Service Monitor

information of each sub-service invocation, in order to calculate the total cost of the whole composed service execution. CSR stores this total cost into the Service Monitor, indicating the performance of the composed service. CSR has also the functionality to log the response time of each individual sub-service invocation and size of the SOAP request/response. From this information, CSR can update the cost information of each individual service instance in the Service Monitor.

\subsection{Service Monitor}

The service monitor (depicted in Figure 7 is storing fastchanging, live and up-to-date data about the currently usable service instances. The storage is realized as a inmemory data structure rather than a database structure for fast access and to allow performing easy updates. Examples of usage include: a service detects that it is malfunctioning and self-reports that fact to the service monitor which generates an alert. Similarly when a device joins the network, the Service Monitor picks up the events from the Discovery component and stores the fact that the services are there.

\subsection{Monitoring and Inventory}

The Monitoring and Inventory is composed of several components: Device Repository, Device Monitor, Middleware Historian, and Discovery.

The Device Repository component is responsible for storing static information about the devices available in the system. To facilitate the registration of numerous devices that have common characteristics, the Device Repository supports the concept of Device Type. The Device Type contains Properties and Features which describes common characteristics of a class of devices. For example, a wireless sensor node such as a SunSPOT (www. sunspotworld. com)can be registered as a Device Type with temperature and acceleration sensors as features. Numerous sensor nodes can be registered using the same device type as reference.

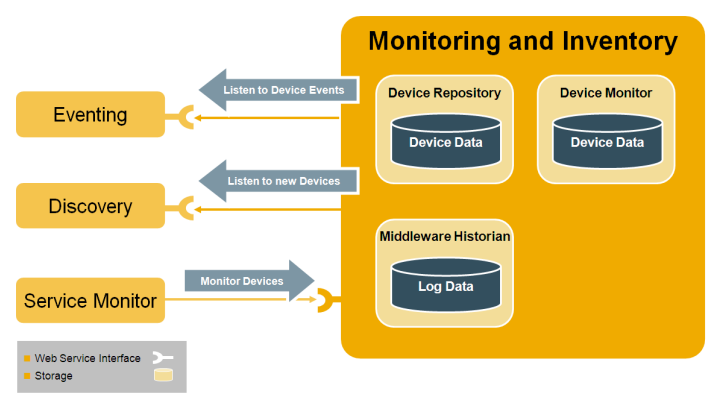

Figure 8. Monitoring and Inventory

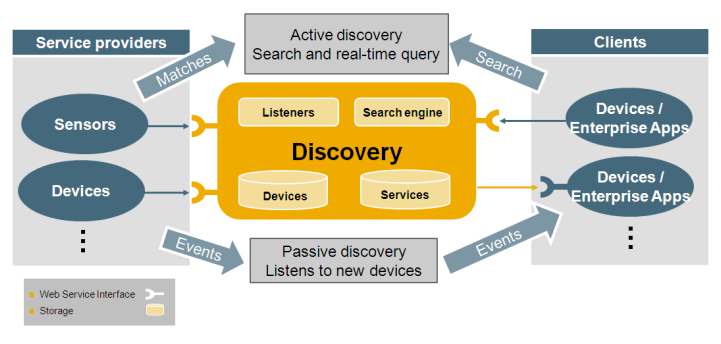

Figure 9. Device Discovery

The Device Monitor monitors devices and detects events like failures. When a malfunction is detected, the component triggers a Fault Event which is propagated to interested parties. As each device has different characteristics and behavior, a set of fault detection techniques is required to monitor devices. These techniques can make use of events generated by the devices, for instance to identify if unexpected value readings were generated. Another possibility is to invoke specific methods on the devices, for instance to ping the device in order to verify if it is still active. New techniques can be used by implementing another monitoring method.

The Middleware Historian acts as an event archive for the architecture. Data from the devices (due to events or invocation), middleware system information, debugging messages, etc. can be stored there and are accessible for correlation of events.

The Discovery component is able to find devices present on a network, and retrieve information about them and their hosted services to be used by other components from the architecture. Two distinct functional modes are implemented by the discovery unit. The first is referred to as "passive discovery", whose role is to listen to the network for announcements from new, unknown devices that connect to the network, dynamically retrieve their metadata and use the eventing mechanism to notify the systems about the devices and their hosted services. The second mode, called "active discovery", is responsible for dynamic search of specific devices or services, and particularly suited for contexts where 


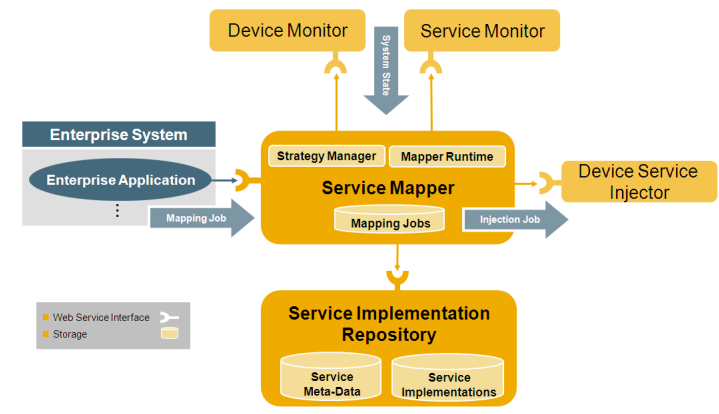

Figure 10. Service Lifecycle Management

new devices with unknown capabilities continuously connect to the system.

\subsection{Service Lifecycle Manager}

The service lifecycle management [4] (depicted in Figure 10) component consists of two main sub-components: the Service Mapper and the Service Implementation Repository. The Service Mapper (SM) performs deployment planning and deployment execution within the SOCRADES middleware. Whenever a business process is created it decides how this process can be represented by the middleware respectively by the services hosted on devices within the device layer of the middleware. The SM therefore generates desired service mappings that describe which services on which devices may be used during execution of a business process (while the actual decision about the used service instance is made by the Service Monitor at runtime). Desired mappings are then transformed into deployment lists, which describe actions to be performed in order to establish a desired mapping. Actions included in a deployment list may be (un)deployment, (de)activation, migration, or configuration of services. The decisions of the SM therefore tremendously influence the reliability of the SOCRADES middleware and thus the reliability of all business processes based on it.

The Service Implementation Repository (SIR) saves all services which are available for composition of new services, orchestration of business process or deployment within the SOCRADES middleware. Saving a service means saving its metadata as wells as associated arbitrary content like service implementations. The SIR is therefore a registry repository rather than only a repository as indicated by its name. The SIR generally offers two kinds of operations related to services which together make up the service lifecycle management of the SIA: management and query. Management refers to the publication of new services, updating existing services (incl. versioning), creation of configurations and deprecation of services. Querying a service may be done using different query languages and

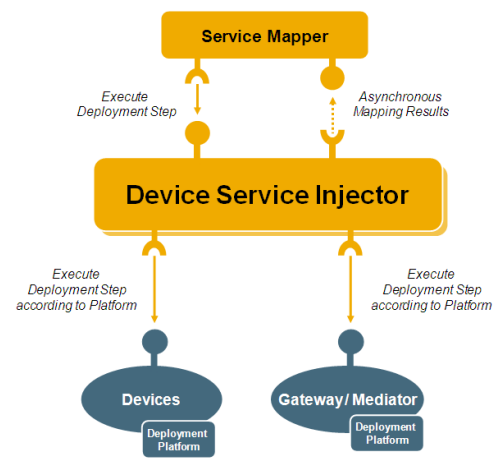

Figure 11. Device Service Injector

constructs and may search for service metadata or associated implementations. All operations may be approved before execution according to rules and guidelines thus enforcing a governance process.

\subsection{Device Service Proxy}

The device service proxy eases the integration of several categories of heterogeneous devices. Plugins can be written that uniformly address devices based on their capabilities. As an example, we have implemented a Devices Profile for Web Services (DPWS) plug-in that can dynamically discover and integrate any DPWS device (such as the ones described in the Demonstration section). Similarly other devices based on OPC-UA, specific RFID or legacy protocols can be integrated provided that a platform plug-in is written for their category.

\subsection{Device Service Injector}

The Device Service Injector (DSI) is responsible for the execution of deployment levels and steps during the processing of a deployment list by the Service Mapper. The DSI utilizes various injectors. For each deployment platform supported by the SOCRADES middleware one injector implementation exists of which various instances during runtime may be started. Each instance registers itself during start up with the DSI and may thus be used for the deployment execution. Injector implementations may either run locally as a subcomponent of the DSI or as remote components possibly on different subnets/locations. This allows the DSI to always choose the most suitable injector currently available for each specific deployment step. Incoming deployment steps are saved within a command queue and are executed either sequentially or in parallel. Delivery of execution results to the Service Mapper may be performed asynchronously. In case errors occur during execution of deployment steps, all steps of the same job are 


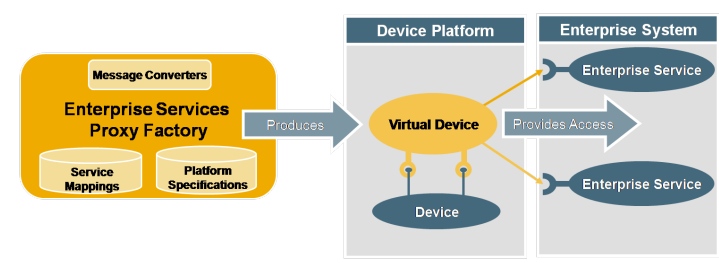

Figure 12. Enterprise Services Proxy Factory

either canceled if they are still in the command queue or compensated if they were already executed.

\subsection{Enterprise Services Proxy Factory}

The Enterprise Services Proxy Factory provides interfaces for the creation of virtual devices that act as intermediaries between the device level and enterprise services. Such proxies might contain a definable set of adapters for enterprise services and provide their respective device interfaces on a target platform. The component defines a set of requirements that need to be fulfilled in order to deploy such virtual devices on a target platform. This includes the installation of platform support on the one hand and one the other hand the formal description of a mapping between device level and enterprise service messages. The Enterprise Services Proxy Factory includes subcomponents that facilitate the process of mapping a device level invocation message to a target enterprise service request and support a customizable set of rules that are applied when converting messages.

\section{Demonstration}

To demonstrate that the aforementioned ideas can be realized, we have used state of the art technologies to implement prototypes. Several prototypes have been implemented as proof of concept.

The systems described have common characteristics where we focus on smart devices like sensors, PLCs or IT devices that offer web service interfaces, either directly or through the use of gateways or service mediators. Through these interfaces they offer functional services (e.g. start/stop, swap to manual/automatic mode) or status information (e.g. power consumption, mode of operation, usage statistics). In our prototypes, the business logic services are supported by a service composition engine and visualized using a visualization toolkit. An operator can use existing tools like SAP Manufacturing Integration and Intelligence (SAP MII) to create the business rules. The SIA enables sophisticated business software such as ERP or PLM systems to interact with the real world. By making web service mash-ups of services running on the enterprise, middleware,

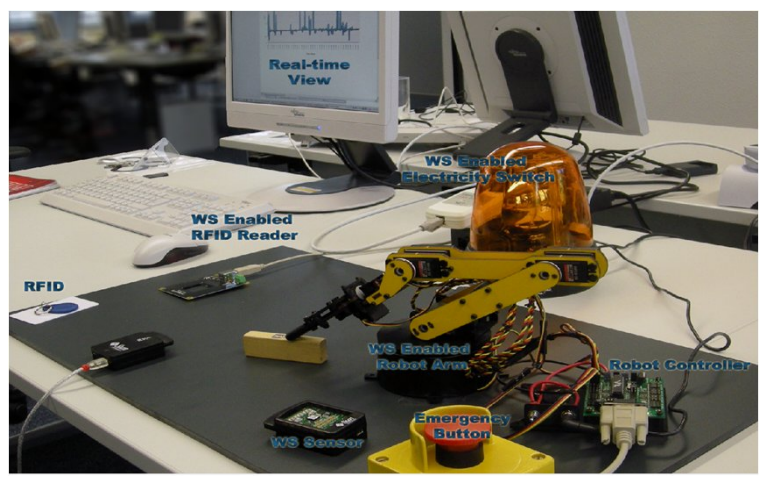

Figure 13. Heterogeneous Web-Service enabled Devices in an Event-based Infrastructure

and device layer, we can provide users with a rich visualization of the real-world status.

The integration of heterogeneous devices is clearly depicted in our latest prototype that successfully shows how several devices supporting web services (or being wrapped by them) can be integrated via the proposed architecture. We have taken several IP devices and wrapped their functionality with web services. More specifically as depicted in Figure 13 we used the following devices: An RFID reader: Appearing RFID tags are read by the reader which raises events showing info with respect to the tag. Each tag is considered to be integrated with a product and serves as a token that links it with business information (e.g. order details). A robotic arm: The functionality (grab, move, etc.) of the robotic arm controller has been wrapped. An electricity IP switch: An alarm lamp has been attached in an electricity IP switch, which offers the on/off functionality as a web service. Wireless sensors: One sensor controls the emergency button. The emergency button has been attached to the IO pins of the SunSPOT wireless sensor. Pressing or releasing the button is captured by the SunSPOT, and an WS-event is generated. Another wireless sensor is used for vibration monitoring. The capability of SunSPOTs to measure acceleration is used for vibration monitoring via the sensor mounted on the robotic arm. This sensor monitors the transportation conditions of the product to make sure it adheres to the product-specific quality guidelines.

Composing application scenarios is dynamically done by orchestrating the available services. The dynamicity also comes from the fact that the SIA is an event-based infrastructure. A typical scenario is the following: As production parts arrive at the factory shop floor they are equipped with an RFID tag. As they reach the processing point, the tag is read by the RFID reader. An event is generated by the reader with all the necessary data like the RFID number and stored on the network. The robot gets notified by this event 


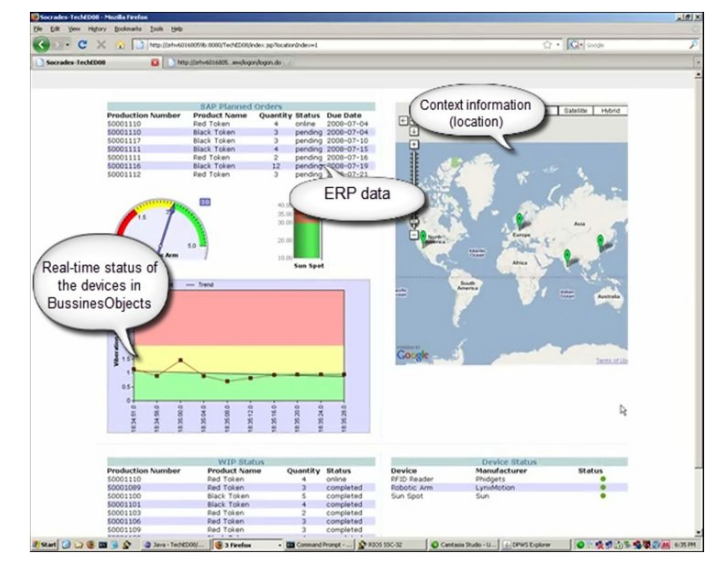

Figure 14. Real time reporting via Enterprise
reporting tools

(as it has subscribed to the service) and picks up the production part. By matching data from the enterprise system and the RFID tag, it knows how to further process the part. In parallel the SunSPOT sensor mounted on the robot monitors the vibration and if it exceeds a specific threshold an event is raised to immediately stop the process (quality control). The same holds true for the emergency switch, if an operator at the shop floor for any reason wants to immediately stop the process (e.g. due to an accident) s/he presses this switch, which is controlled by a SunSPOT that again generates an event.

Once such an emergency event is propagated, devices that consume it react accordingly. The robot receives the emergency shutdown event and immediately stops its operation. Also the IP electricity switch, once it receives the event, turns on the emergency light. At business layer, there is an enterprise application such as shop-floor reporting realized by the SAP product Business Objects. There, we have a real-time monitoring of the shop-floor status as the application also subscribes to all events raised. The plant manager can immediately see the status of the ERP orders (Figure 14), the production progress, the device status, as well as a global view on all factories and the possible sideeffects of a production line delay due to shop-floor device malfunctions.

All of the communication is done via web service technologies and the realization of this specific scenario became possible by having a composition of the available functionality that all devices and applications expose as a service. In legacy systems, integration of a new device or reassignment of its role would result in reconsidering how the device integrates with other devices and how they control it. However with the SOA approach described, the implementation of a new scenario is possible by modifying the orchestration of the services already available.
An online demo of the Integration Architecture is available at http://socrades.dyndns.org:50000/SIA_ demo_web/demo-intro.html.

\section{Conclusion and Future Work}

We have presented the design of an architecture that can strongly couple the envisioned Internet of Things infrastructure with enterprise services. The majority of the specified functionality has been implemented and trialed.

The approach is event-based and its interaction with external entities as well as internal communication is based on web services. Theoretically each component could be hosted in different locations. As an example, parts of the architecture could be running in the cloud offering high-performance services, while parts interacting locally with the devices could run even on embedded systems located at the device layer.

The design and implementation was done with scalability in mind. However, we plan to conduct extended scalability and performance tests in the future. A special focus will be on performance tests when running DPWS on resource-constrained devices. Future work will also include the extension of the architecture to support additional devices using OPC-UA and REST plug-ins. Validation trials with real-world devices are planned in the automation and energy domain.

\section{Acknowledgments}

The authors would like to thank the European Commission and the partners of the European IST FP6 project Service-Oriented Cross-layer infRAstructure for Distributed smart Embedded devices SOCRADES (www. socrades. eu), for their support.

\section{References}

[1] L. M. S. de Souza, P. Spiess, D. Guinard, M. Koehler, S. Karnouskos, and D. Savio. Socrades: A web service based shop floor integration infrastructure. In Proc. of the Internet of Things (IOT 2008). Springer, 2008.

[2] W. K. Edwards. Discovery systems in ubiquitous computing. IEEE Pervasive Computing, 5(2):7077, 2006.

[3] E. Fleisch and F. Mattern. Das Internet der Dinge. Springer, 1 edition, July 2005.

[4] T. Frenken, P. Spiess, and J. Anke. A Flexible and Extensible Architecture for Device-Level Service Deployment, volume 5377 of LNCS, pages 230-241. Springer, December 2008.

[5] F. Jammes and H. Smit. Service-oriented paradigms in industrial automation. IEEE Transactions on Industrial Informatics, 1(1):62-70, Feb. 2005.

[6] S. Karnouskos, O. Baecker, L. M. S. de Souza, and P. Spiess. Integration of soa-ready networked embedded devices in enterprise systems via a cross-layered web service infrastructure. In Proc. of the 12th International Conference on Emerging Technologies \& Factory Automation (ETFA), pages 293300, 25-28 Sept. 2007. 